\title{
SOBRE VARIAÇÕES NA TÉCNICA DE TRICRÔMICO DE GOMORI PARA ESTUDO DE HELMINTOS DA CLASSE MONOGENOIDEA E FAMÍLIA DACTYLOGYRIDAE
}

\author{
Anderson da Costa de Almeida Silveira \\ Graduando em Biologia/Faculdades São José (FSJ)/RJ \\ andersonosredna@gmail.com
}

Karina da Silva Silveira de Almeida

Especialista em Ictioparasitologia pelo Programa de Capacitação Profissional em Serviço/Instituto

Oswaldo Cruz (FIOCRUZ)/RJ

silveirakarina89@gmail.com

\section{RESUMO}

Os Monogenoidea são considerados importante objeto de estudo da Ictioparasitologia, pois estão entre os grupos mais numerosos e que causam os maiores danos aos peixes. Entretanto, ainda tendo ganhado maior importância ao longo dos anos, a Ictioparasitologia conta com poucos profissionais aptos a realizarem os procedimentos que permitem a identificação dos parasitos, e esses estudos são cruciais, principalmente para determinar maneiras de combater e controlar estes parasitos, sobretudo em peixes cultivados por práticas de piscicultura e aquicultura. Assim sendo, o presente trabalho tem como principal objetivo recomendar algumas variações em um importante protocolo de técnica utilizado para estudo da morfologia interna dos Monogenoidea, a fim de facilitar a execução do passo-a-passo. Após sucessivas repetições, as variações propostas geraram resultados satisfatórios, e os espécimes corados foram utilizados em trabalhos taxonômicos, sendo identificados inclusive com captura de imagens através de fotografias, que estão inclusas em trabalhos submetidos à publicação em outras revistas científicas.

Palavras-chave: Monogenoidea; Protocolos e técnicas laboratoriais; Morfologia interna.

\begin{abstract}
The Monogenoidea are considered important object of study of Ictioparasitologia because they are among the most numerous and cause the greatest damage to the fish groups. However, having also gained increased importance over the years has few Ictioparasitologia able to perform the procedures that allow the identification of parasites professionals, and these studies are crucial, especially to determine ways to combat and control these parasites, especially in fish cultivated for farming and aquaculture practices. Therefore, this study aims to recommend some changes in protocol an important technique used to study the internal morphology of Monogenoidea in order to facilitate the execution of the step-by-step. After successive repetitions, the proposed changes generated satisfactory results, and stained specimens were used in taxonomic work, including being identified image capture through photographs, which are included in papers submitted for publication in other journals.
\end{abstract}

Keywords: Monogenoidea; Protocols and laboratory techniques; Internal morphology.

Persp. online: biol. \& saúde, Campos dos Goytacazes, 12 (4), 1-7, 2014 


\section{INTRODUÇÃO}

O estudo dos parasitos de peixes é denominado Ictioparasitologia (LUQUE, 2004). Nos últimos anos, estes mesmos estudos vêm ganhando força por diversos motivos. Dentre estes, citamos o monitoramento da qualidade das águas, uma vez que a maioria destes parasitos faz parte do zooplâncton, que é muito sensível às modificações sofridas nos ambientes aquáticos (THATCHER \& BRITES-NETO, 1994). Estudos relacionados à Ictioparasitologia também vêm gerando subsídios para monitoramento e controle de patogenias graves causadas em peixes cultivados através de práticas de piscicultura e aquicultura (ALMEIDA \& COHEN, 2011).

Como exemplo importante, destaca-se o ocorrido no rio Curuá-Una, no Estado do Pará, onde após o represamento deste rio para o início das atividades da Usina Hidrelétrica Curuá-Una, dentre os impactos causados, constatou-se infestação maciça de peixes com parasitas. Alguns anos depois do represamento, os peixes capturados no reservatório apresentaram uma elevada taxa de infestação com nematódeos, o que fez com que a população local praticamente parasse de pescar e consumisse peixe (JUNK \& MELLO, 1990).

\subsection{A classe Monogenoidea}

Os Monogenoidea ou Monogenea (reino Animalia, filo Platyhelminthes), estão entre os mais numerosos e importantes grupos de parasitos de peixes. Eiras (1994), afirma que existem aproximadamente 10.000 espécies de parasitos de peixes distribuídos entre sete grupos zoológicos. Os Monogenoidea estão em segundo lugar, com 1.500 espécies descritas, enquanto que Protozoa está em primeiro, com 1.750 espécies descritas.

Monogenóideos são ectoparasitos hermafroditos, com ciclo de vida direto (monoxênico), ou seja, completam seus estágios de vida (ovo, oncomiracídio, adulto) em um único hospedeiro, no caso, os peixes, exibindo alta especificidade a eles (BYCHOWSKY, 1957; KRITSKY et al., 1997).

Dentre as estruturas mais importantes do parasito, destaca-se o haptor (ou "opisthaptor"), principal órgão de fixação destes parasitos, formado por estruturas esclerotizadas em formato de grampos ou ganchos, que também possui importância taxonômica (CRIBB et al., 2002).

As espécies parasitas de Monogenoidea pertencem a duas principais famílias: Dactylogyridae e Gyrodactylidae. Os girodactilídeos são em maioria vivíparos, parasitas de tegumento (LUPCHINSKI Jr et al., 2006). Entretanto, na América do Sul os dactilogirídeos são os mais numerosos. Eles são ovíparos, encontrados principalmente nas brânquias de peixes (BOEGER \& VIANNA, 2006).

Segundo Poulin (2002) a maior parte dos dactilogirídeos mede menos de um milímetro de comprimento. Geralmente possuem o corpo alongado e ligeiramente achatado dorso-ventralmente. Os adultos possuem dois pares de manchas ocelares que podem estar dispersos na forma de grânulos, entretanto podem estar ausentes em algumas espécies. Essas estruturas ficam localizadas em frente e por cima da faringe. Na parte anterior do corpo possuem dois lobos cefálicos com células que secretam substância adesiva, com função de "amolecer" os tecidos do hospedeiro, facilitando a nutrição do parasito. O pênis ou órgão copulatório masculino (OCM) é esclerotizado, também sendo considerado muito importante em estudos taxonômicos para identificação das espécies deste grupo de parasitos. Apresentam ainda faringe muscular e intestino posterior tubular. O haptor é geralmente formado por dois pares de ganchos centrais ou âncoras, que são unidos por duas barras transversais, uma ventral e outra dorsal, além de 14 ganchos marginais (EIRAS, 1994; KEARN, 1994).

Geralmente estes parasitos se nutrem de sangue, muco e tecidos de seus hospedeiros, e podem provocar patogenias graves. Nas infestações menos intensas causam apenas feridas e ulcerações que ocasionalmente servem como porta de entrada para infecções secundárias causadas por vírus e bactérias, por exemplo. Já em casos mais graves, podem levar seus hospedeiros à morte por asfixia, uma vez que sua 
presença nas brânquias causa aumento da produção do muco, o que promove a união dos filamentos branquiais, impedindo as trocas gasosas (MARTINS \& ROMERO, 1996; EIRAS, et al., 2000; PAVANELLI et al., 2002; BOEGER \& VIANNA, 2006).

\subsection{Estudos em Ictioparasitologia}

Existem espécies com grande importância econômica, principalmente na família Dactylogyridae, que vêm gerando grandes prejuízos para a área da piscicultura no Brasil. Desta forma, o estudo adequado e intensificado destes parasitos é fundamental. Assim sendo, a preparação qualificada dos espécimes também é importantíssima. Geralmente, os estudos taxonômicos em monogenéticos costumam centrar-se mais nas partes esclerotizadas, principalmente no haptor (CRIBB et al., 2002). Entretanto, estudos relacionados com os órgãos e estruturas internas (ovário, testículos, esôfago, vitelinos) também são bastante relevantes e muitas vezes cruciais para a complementação dos estudos das partes esclerotizadas (BOEGER \& VIANNA, 2006).

Whittington (1998) afirma que a fauna íctica mundial é parasitada por aproximadamente 25.000 espécies de monogenóideos, entretanto até o momento só existem de 3.000-4.000 descritas. A ocorrência destes fatos está relacionada intimamente ao fato de existirem poucos taxonomistas aptos para realizarem os procedimentos de identificação de espécies (BROOKS, 2000). Diante disso, podemos perceber que um estudo morfológico adequado e completo de helmintos da classe Monogenoidea não só exige a preparação de espécimes para a observação das partes quitinosas, mas também dos órgãos internos, que não são quitinosos. A escolha do corante costuma ser muito importante e definitiva para a preparação de um bom material para estudo da morfologia interna dos Monogenoidea. O tricrômico de Gomori é o corante mais utilizado, com melhores resultados para este fim (BOEGER \& VIANNA, 2006).

Diante do exposto, o objetivo principal deste trabalho é propor algumas recomendações no protocolo de técnicas utilizadas para o estudo da morfologia interna de monogenóideos da família Dactylogyridae, utilizando-se como base o protocolo proposto por Boeger \& Vianna (2006), a fim de facilitar a execução do passo-a-passo.

\section{METODOLOGIA}

A técnica foi adaptada a partir de várias repetições do protocolo proposto por Boeger e Vianna (2006). Durante os trabalhos de Almeida (2011) e Almeida (2012), a mesma foi sendo variada de acordo com repetições e observações, anotando as variações realizadas após verificação do sucesso alcançado.

Primeiramente, na técnica de tricrômico de Gomori, conforme ressaltado por Almeida \& Cohen (2011) devem ser selecionados os parasitos mais "intactos e "retos", pois nesta técnica é possível observar os órgãos internos do parasito, e se o mesmo estiver com muitas contrações e torções corporais, não será possível a visualização destes órgãos. No momento do procedimento de coloração, no caso dos dactilogirídeos, o ideal é não permitir que o espécime escape do pincel (utilizar pincel de número 0, sendo necessário ainda cortar e retirar algumas cerdas, pois o parasito é bem pequeno) para a placa com os conteúdos do passo-a-passo, de preferência. Sobre isso, na etapa do álcool absoluto, é um tanto quanto difícil permitir que o parasito fique no pincel, por isso é necessário utilizar pouca quantidade do mesmo.

Também é importante ressaltar que se deve usar pouca quantidade de água, tricrômico e creosoto de Faia, além do próprio álcool absoluto. A água deve-se usar três gotas, no máximo; o tricrômico, apenas uma gota; álcool, dez gotas e creosoto, duas gotas. A água, o álcool e o creosoto são importantes repor de acordo com a evaporação. O tricrômico deve ser reposto quando o mesmo começa a se solidificar. Isso pode ser observado quando o corante começa a parecer endurecido nas bordas da gota utilizada no processo.

Ainda sobre a técnica, pode-se dizer que não foi necessário, nas etapas de tricrômico e creosoto de Faia, que o parasito fosse colocado dentro de uma placa com os conteúdos descritos no passo-a-passo. Em 
alguns casos, o tricrômico de Gomori e o creosoto estiveram colocados em uma lâmina - numa extremidade a gota de tricrômico e na outra o creosoto de Faia. Sobretudo no momento de corar o espécime com tricrômico, afirma-se que o manuseio e apoio do pincel às mãos se tornaram mais fáceis, uma vez que basta apenas encostar o parasito no corante, sem precisar que o mesmo fique submerso. Com as bordas da placa de Petri, tanto o apoio do pincel como das mãos fica comprometido.

\subsection{Passo-a-passo}

1- Transferir até 10 parasitos da formalina para uma placa de Petri contendo duas a três gotas de água (podendo eles ficar nesta mesma gota durante bastante tempo), diferentemente do protocolo de Boeger \& Vianna (2006), onde se deve colocar de 1 a 5 parasitos diretamente em tricrômico de Gomori.

2- Transferir um parasito (a partir desta etapa, trabalhar apenas com um parasito por vez, até a etapa de número 4) para uma placa contendo uma gota de tricrômico de Gomori. Deve-se encostar o espécime com o pincel no corante com cautela, sem permitir, de preferência, que o mesmo escape e se perca na gota de corante. Dessa forma, é possível verificar que o espécime absorve o corante por capilaridade. No caso de dactilogirídeos do gênero Tereancistrum (ALMEIDA, 2012), os mesmos são extremamente pequenos, ao ponto de a análise do material em microscópio estereoscópico ter de ser feita várias vezes. A variação no protocolo foi utilizada, e o resultado foi bastante satisfatório, sendo possível, principalmente em exemplares do gênero Cosmetocleithrum (ALMEIDA, 2011), observar vários órgãos internos (gônadas, vitelinos, órgãos cefálicos). No protocolo original, a indicação é que os parasitos deveriam ser corados por 1 a 5 minutos em tricrômico de Gomori, dependendo do tamanho dos espécimes.

3- Transferir o parasito para uma placa com álcool absoluto observando, até que, como descrito por Boeger \& Vianna (2006), o mesênquima esteja levemente corado (geralmente verde claro). Algumas vezes, foi possível observar que o espécime perdeu coloração além da conta, e foi necessário retornar à etapa do tricrômico, entretanto não houve nenhum dano a ele. Posteriormente, o espécime foi novamente colocado no álcool absoluto. Na orientação de Boeger \& Vianna (2006), mais de um parasito deveriam ficar na mesma placa, onde deveria ser adicionado álcool absoluto e água, para que ocorresse a diferenciação, que seria a remoção do excesso de corante dos espécimes de modo que o mesênquima ficasse com coloração verde clara. Depois disso, a placa deveria ser agitada, para ocorrer a total mistura da água com o álcool absoluto.

4- Caso o parasito fique com cores mescladas entre o verde claro e azul escuro, mesmo após passar pela etapa do álcool absoluto, encostar o mesmo com o pincel em algumas poucas gotas de álcool 70\%, com cuidado para que o mesmo não perca muito a coloração. Caso isso ocorra, retornar para a etapa do tricrômico.

5- Transferir o parasito rapidamente para uma placa com uma gota de creosoto de Faia para clarificar (os parasitos corados ficaram longos períodos de tempo no creosoto, aproximadamente 15 minutos, sem maiores problemas para a preservação do mesmo). Boeger \& Vianna (2006) indicam que deveria ser colocada uma gota de creosoto de Faia em uma lâmina, onde apenas um parasito deveria ser posto. Logo após, o excesso de creosoto de Faia deveria ser removido com lenço de papel e, nessa mesma lâmina, deveria ser colocada uma gota de bálsamo do Canadá, e para finalizar, recobrir a lâmina contendo o espécime com uma lamínula. a lamínula.

6- Transferir o parasito para uma lâmina contendo uma gota de bálsamo do Canadá, recobrindo com

As espécies utilizadas nos procedimentos realizados neste trabalho foram pertencentes ao gênero Cosmetocleithrum e Tereancistrum, e uma pertencente à família Dactylogyridae, que ainda não foi identificada. Todas estas espécies são parasitas de Trachelyopterus striatulus (Steindachner, 1877) (Cosmetocleithrum) e Brycon pesu (Müller \& Troschel, 1845) (Tereancistrum e Dactylogyridae não identificada). 
Foram confeccionadas exatamente 86 lâminas de espécimes do gênero Cosmetocleithrum preparados pela coloração com tricrômico, de um total de 644 espécimes coletados (ALMEIDA, 2011).

Nos espécimes do gênero Tereancistrum e Dactylogyridae não identificada, de um total de 39, 11 foram preparados com a técnica de coloração por tricrômico de Gomori (ALMEIDA, 2012).

Todos os espécimes de Monogenoidea foram coletados em peixes provenientes dos rios Guandu (RJ), no caso de T. striatulus e Tocantins (MA), em B. pesu.

Quanto à determinação exata das espécies de Monogenoidea às quais as lâminas de tricrômico confeccionadas pertencem, é necessário que as análises taxonômicas sejam primeiramente encerradas, fato que ainda não ocorreu.

O corante utilizado no presente estudo foi adquirido pronto, fabricado a partir da fórmula indicada em Boeger \& Vianna (2006) - Cromotropo 2R (C.I. 16570): 0,6 g; Azul anilina: 0,6 g; Ácido fosfomolibdênico: $1 \mathrm{~g}$; dissolver a mistura em água destilada: $100 \mathrm{ml}$; Adicionar ácido clorídrico: $1 \mathrm{ml}$. Guardar na geladeira por 24 horas antes de usar. Manter refrigerado e usar frio.

\section{RESULTADOS E DISCUSSÃO}

Com as variações propostas na técnica, os exemplares preparados apresentaram boas condições de estudo, inclusive com descrição taxonômica dos mesmos. Em Almeida (2011), o número de exemplares envolvidos no estudo foi grande, dentre os quais se separaram inicialmente 15 adequados para $o$ procedimento, que foram preparados sem as variações na técnica, e os mesmos foram todos perdidos, nem mesmo chegaram a ser confeccionadas lâminas, já que todos se desintegraram. O mesmo ocorreu em Almeida \& Cohen (2011), onde inicialmente foram obtidos 20 exemplares adequados, e os 20 foram perdidos.

Deste modo, as variações começaram a ser realizadas justamente em Almeida (2011), após verificar a perda excessiva de exemplares corados. Com a realização das variações, foram confeccionadas 86 lâminas de tricrômico de Gomori, o que correspondeu a $13 \%$ de todo o material obtido para estudo, uma vez que também é importante considerar que deve haver a escolha dos exemplares adequados para o procedimento da coloração, que são aqueles que não apresentam torções corporais. Em Almeida (2012), as variações na técnica também foram utilizadas, obtendo-se $28 \%$ do material com exemplares corados em tricrômico de Gomori, pois também só esta quantidade estava apropriada para o procedimento.

Dessa forma, com relação a este procedimento, não foi proposto um novo protocolo, apenas foram recomendadas algumas práticas para justamente facilitar a execução do passo-a-passo com a preparação de dactilogirídeos para o estudo da morfologia interna, como inclusive foi recomendado por Boeger \& Vianna (2006), que orientam que o mais prudente seria testar o tempo em que o parasito deve ficar no corante, pois o mesmo varia de acordo com o tamanho dos espécimes. Portanto, verificamos neste trabalho que este tempo realmente varia, e que a melhor solução encontrada para o tempo de coloração (principalmente em dactilogirídeos dos gêneros Tereancistrum e Cosmetocleithrum) foi somente encostar o parasito no corante, sem precisar que o mesmo fosse submerso.

\section{CONCLUSÕES}

Com os espécimes obtidos nos trabalhos de Almeida (2011) e Almeida (2012), foi possível concluir que as variações propostas no passo-a-passo de Boeger \& Vianna (2006) obtiveram resultados satisfatórios, justamente porque os parasitos corados foram utilizados para fins taxonômicos com sucesso, e seguem envolvidos em estudos onde as lâminas preparadas estão sendo analisadas para determinar as espécies às quais os exemplares corados pertencem. Principalmente no trabalho de Almeida (2011), também foram feitas 
fotografias dos espécimes corados, e estas mesmas fotografias já estão sendo inclusas em trabalhos relacionados à área de taxonomia, que estão submetidos para publicação em outros periódicos científicos.

\section{REFERÊNCIAS BIBLIOGRÁFICAS}

ALMEIDA, A. C. Monogenea, Digenea e Nematoda parasitos de Trachelyopterus striatulus provenientes do Rio Guandu, Estado do Rio de Janeiro. Monografia de conclusão de curso. Graduação em Ciências Biológicas das Faculdades São José, 2011.

ALMEIDA, K. S. S. Técnicas de coleta e processamento para o estudo da morfologia, taxonomia e biologia de Monogenoidea parasitos de Brycon pesu (Müller \& Troschel, 1845) (Characiformes: Characidae) provenientes do trecho médio do rio Tocantins, divisa dos estados do Maranhão e Tocantins. Monografia de conclusão de curso de especialização em Morfologia, Taxonomia e Biologia de Monogenoidea. Programa de pós-graduação latu senso em Capacitação Profissional em Serviço do Instituto Oswaldo Cruz - FIOCRUZ, 2012.

ALMEIDA, K. S. S. \& COHEN, S. C. Diversidade de Monogenea parasitos de Astyanax altiparanae da Usina Hidrelétrica de Itaipu. Saúde e Ambiente em Revista, vol. 6, n. 1, p. 31-41, 2011.

BOEGER, W. A. \& VIANNA, R. T. Monogenoidea. In: THATCHER, V. E. (ed.) Amazon Fish Parasites. Pensoft Publishers, Sofia, p. 42-116, 2006.

BROOKS, D. R. Parasite systematics in the 21 st century: opportunities and obstacles. Memórias do Instituto Oswaldo Cruz, vol. 95, suppl. 1, p. 99-107, 2000.

BYCHOWSKY, B. E. Monogenetic Trematodes. Their Systematic and Phylogeny. Moscow: Academy of Sciences, 1957.

CRIBB, T. H.; CHISHOLM, L. A. \& BRAY, R. A. Diversity in the Monogenea and Digenea: does lifestyle matter? International Journal for Parasitology, vol. 32, n. 3, p. 321-328, 2002.

EIRAS, J. C. Elementos de Ictioparasitologia. Fundação Engenheiro Antônio de Almeida, Porto: Edições Afrontamento, 339p., 1994.

EIRAS, J. C., TAKEMOTO, R. M. \& PAVANELLI, G. C. Métodos de estudo e técnicas laboratoriais em parasitologia de peixes. Maringá: Editora Universidade Estadual de Maringá, 171p., 2000.

JUNK, W. J. \& MELLO, J. A. S. N. Impactos ecológicos das represas hidrelétricas na bacia amazônica brasileira. Estudos Avançados, vol. 4, n. 8, p. 126-143, 1990.

KEARN, G. C. Evolutionary expansion of the Monogenea. International Journal for Parasitology, vol. 24, n. 8, p. 1227- 1271, 1994. 
KRITSKY, D. C.; BOEGER, W. A. \& JÉGU, E. M. Neotropical Monogenoidea. 29. Ancyrocephalinae (Dactylogyridae) of piranha and their relatives (Teleostei, Serrasalmidae) from Brazil: species of Amphitecium Boeger and Kritsky, 1988, Heterothecium gen. n. and Pithanothecium gen. n.. Journal of the Helminthological Society of Washington, vol. 63, p. 153-175, 1997.

LUPCHINSKI - Jr., E.; VARGAS, L.; RIBEIRO, R. P.; MOREIRA, H. L. M.; VALENTIM, M.; POVH, J. A. A importância da utilização da técnica RAPD para a identificação de dactilogirídeos em Tilápias do Nilo (Oreochromis niloticus). Arquivo de Ciências Veterinárias e Zoologia da UNIPAR, vol. 9, n. 1, p. 49-57, 2006.

LUQUE, J. L. Biologia, Epidemiologia e Controle de Parasitos de Peixes. Revista Brasileira de Parasitologia Veterinária, vol. 13, n. 1, p. 161-165, 2004.

MARTINS, M. L. \& ROMERO, N. G. Efectos del parasitismo sobre el tejido branquial em peces cultivados: estudio parasitológico e histopatológico. Revista Brasileira de Zoologia, vol. 13, n. 2, p. 489-500, 1996.

PAVAnelli, G. C.; EIRAS, J. C. \& TAKEMOTO, R. M. Doenças de peixes. Profilaxia, diagnóstico e tratamento. Universidade Estadual de Maringá, 305p., 2002.

POULIN, R. The evolution of monogenean diversity. International Journal for Parasitology, vol. 32, p. 245-254, 2002.

THATCHER, V. E. \& BRITES-NETO, J. Diagnóstico, prevenção e tratamento das enfermidades de peixes neotropicais de água doce. Revista Brasileira de Medicina Veterinária, vol. 16, p. 111-128, 1994.

WHITTINGTON, I. D. Diversity "down under": monogeneans in the Antipodes (Australia) with a prediction of monogenean biodiversity worldwide. International Journal for Parasitology, vol. 28, n. 10, p. 14811493, 1998. 